\title{
Large-Area Resonance-Tuned Metasurfaces for On-Demand Enhanced Spectroscopy
}

\author{
Masanobu Iwanaga, ${ }^{1}$ Bongseok Choi, ${ }^{1}$ Hideki T. Miyazaki, ${ }^{1}$ \\ Yoshimasa Sugimoto, ${ }^{1}$ and Kazuaki Sakoda ${ }^{1,2}$ \\ ${ }^{1}$ National Institute for Materials Science (NIMS), 1-1 Namiki, Tsukuba 305-0044, Japan \\ ${ }^{2}$ Graduate School of Pure and Applied Sciences, Tsukuba University, 1-1-1 Tennodai, Tsukuba 305-8571, Japan
}

Correspondence should be addressed to Masanobu Iwanaga; iwanaga.masanobu@nims.go.jp

Received 20 January 2015; Accepted 17 February 2015

Academic Editor: Antonios Kelarakis

Copyright (C) 2015 Masanobu Iwanaga et al. This is an open access article distributed under the Creative Commons Attribution License, which permits unrestricted use, distribution, and reproduction in any medium, provided the original work is properly cited.

\begin{abstract}
We show an effective procedure for lateral structure tuning in nanoimprint lithography (NIL) that has been developed as a vertical top-down method fabricating large-area nanopatterns. The procedure was applied to optical resonance tuning in stacked complementary (SC) metasurfaces based on silicon-on-insulator (SOI) substrates and was found to realize structure tuning at $\mathrm{nm}$ precision using only one mold in the NIL process. The structure tuning enabled us to obtain fine tuning of the optical resonances, offering cost-effective, high-throughput, and high-precision nanofabrication. We also demonstrate that the tuned optical resonances selectively and significantly enhance fluorescence (FL) of dye molecules in a near-infrared range. FL intensity on a SC metasurface was found to be more than 450-fold larger than the FL intensity on flat Au film on base SOI substrate.
\end{abstract}

\section{Introduction}

Artificial nanostructures called metamaterials [1-5] and metasurfaces are forming a rapidly developing group of nanomaterials. Recently, novel optical functions for ray control were realized by use of single-layer metasurfaces of complex unit cell [6] and stimulated great interest in metasurfaces. There are two general aspects of metasurfaces: one is to control optical and spectral features with artificial nanostructures of subwavelength thickness and the other is to incorporate resonant effect on the thin structures. To exploit the two aspects, metallic nanostructures are highly useful because plasmonic resonances are efficiently induced in the very thin structures. In comparison with three-dimensional (3D) structures, the effort in fabrication of metasurfaces is much lower, which is also an advantage of metasurfaces.

Fluorescence (FL) enhancement on metallic nanostructures has been extensively investigated for a few decades $[7,8]$. Although large FL enhancement has been expected since the initial studies, significant enhancement exceeding 100 -fold had not been obtained until recently $[9,10]$. This is probably because metal-induced quenching of FL takes place in some configurations $[11,12]$ and nonoptimized metallic nanostructures were used. It is thus expected that metasurfaces contribute to the quest for large FL enhancement.

Nanoimprint lithography (NIL) has been developed for 20 years $[13,14]$ and is currently recognized as an established nanofabrication method with large-area, high-throughput, and high-precision features. NIL has been employed for nanofabrication of electronic and photonic structures [15] and for parallel production of a large number of functional elements [16]. Even high-density nanostructures more than a teradot/inch ${ }^{2}$ have been successfully produced $[17,18]$.

In this paper, we introduce lateral structure tuning in NIL with only one mold and show the fabricated metasurfaces and the optical properties. Resonances on the metasurfaces were applied to enhance FL signals in a near-infrared range and turned out to be well-suited for FL enhancement. We also address spectroscopic features of the metasurfaces, study the resonant electromagnetic (EM) fields by numerical calculations, and discuss practical potential for on-demand enhanced spectroscopy. 


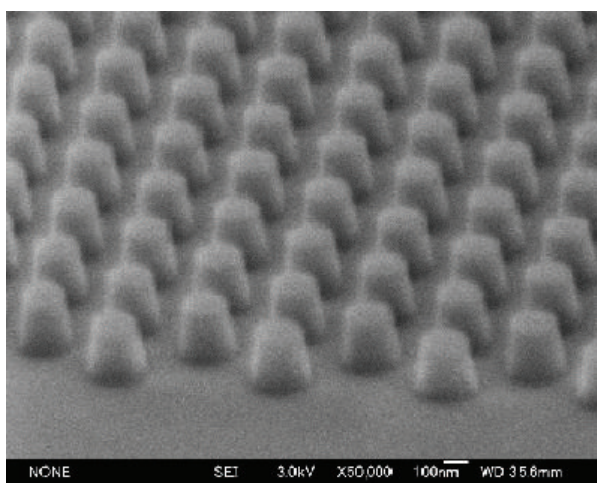

(a)

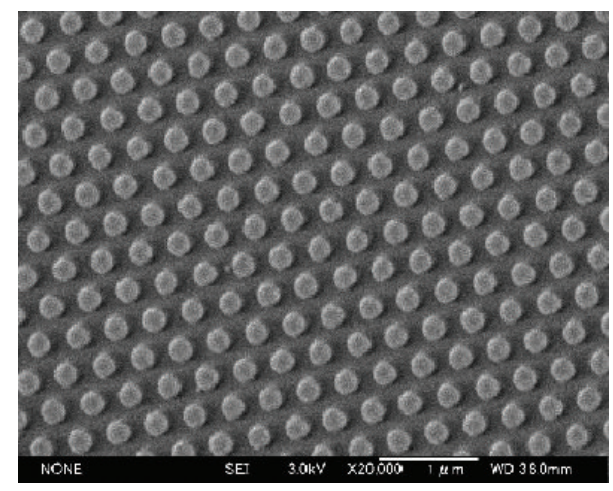

(b)

FIGURE 1: SEM images of a quartz mold of hexagonal array of pillars. (a) Oblique view: white scale indicates $100 \mathrm{~nm}$. (b) Top view: white scale indicates $1 \mu \mathrm{m}$. The periodicity is $410.5 \mathrm{~nm}$ and the diameter of pillars is $200 \mathrm{~nm}$ at the top.

\section{Method}

2.1. Fabrication Procedure. Ultraviolet (UV) NIL procedures were as follows. Resist for UV irradiation was spin-coated on substrates and prebaked in advance. Antiadhesion treatment for a quartz mold was also performed in advance. The quartz mold had hexagonal array of pillars as shown in Figure 1; the periodicity was set to $410.5 \mathrm{~nm}$ and the diameter of the pillars was $200 \mathrm{~nm}$ at the top. A large-area $\left(1 \times 1 \mathrm{~cm}^{2}\right)$ of the hexagonal array was produced on a quartz substrate of $1 \mathrm{~mm}$ thickness by electron-beam lithography. The quartz mold was pressed down to the resist-coated substrate and UV irradiation at $365 \mathrm{~nm}$ was carried out. After removing the mold, the pattern on the quartz mold was transferred to the resist. This situation is illustrated in Figure 2. A thin residual film remained at the bottom of the resist. Typically, the thickness of the residual film was a few tens of $\mathrm{nm}$. It is necessary to remove the residual film. Although the removal is often done with $\mathrm{O}_{2}$ gas plasma, we used mixed-gas plasma of $\mathrm{O}_{2}$ and $\mathrm{N}_{2}$ because we chose to make the etching rate of the resist slower. As a result, we were able to precisely control the size of the resist aperture, which is referred to as $w_{1}$ and $w_{2}$ in Figure 2. This was the key step for lateral structure tuning in the present NIL. After removing the residual thin film, silicon-on-insulator (SOI) layer of $200 \mathrm{~nm}$ thickness was vertically etched down to buried oxide (BOX) layer through Bosch process with $\mathrm{SF}_{6}$ and $\mathrm{C}_{4} \mathrm{~F}_{8}$ gas. The $\mathrm{BOX}$ is equivalent to $\mathrm{SiO}_{2}$. The diameter of air holes was inherited from that of the resist aperture. Further detailed conditions such as specific parameters in the UV NIL were previously reported [19].

To obtain metasurfaces for the present purpose, we conducted a one-time Au evaporation. The thickness of the $\mathrm{Au}$ was $35 \mathrm{~nm}$. A 3D-view schematic of the stacked complementary (SC) metasurface is illustrated in Figure 3(b). The SC metasurface consists of three periodic layers; the top layer is periodically perforated $\mathrm{Au}$ film, the middle layer is a Si slab perforated similarly to the top layer, and the bottom layer comprises a Si slab that includes embedded Au disks. The metallic nanostructures in the top and bottom layers are complementary to each other; accordingly, we call the three-layer stacking structure SC structure. We mainly consider the SC structure at the near-infrared range in this paper. The total thickness of the SC structure was $235 \mathrm{~nm}$ and was in subwavelength range to the wavelengths of present interest; we also consider macroscopic optical response such as reflectance of the SC structure. We thus call the SC structure SC metasurface.

The structures of the fabricated SC metasurfaces were confirmed by scanning-electron-microscope (SEM) images. The top-view SEM images are shown in Figure 3(a); white scale bars indicate $1 \mu \mathrm{m}$. After the Au evaporation, reflectance spectra of the SC metasurfaces in Figure 3(c) were measured. The SOI substrate we employed included BOX layer of $400 \mathrm{~nm}$ thickness and Si wafer of approximately $600 \mu \mathrm{m}$ thickness.

2.2. Optical Measurement. Reflectance spectra were measured using a spectrometer. The incident angle was set to $5^{\circ}$, which was almost equivalent to the normal incidence in the spectral shape. Incident polarization was set to a linear polarization; in the optical configuration of Figure 3(b), the incidence was $p$-polarized; that is, the incident polarization was parallel to the plane of incidence, which was set to be the $x z$ plane.

FL measurement was conducted in a microscopy setup including an objective lens $(\times 20)$ of numerical aperture 0.4 that was set in the normal incidence configuration. Incidence was focused by the objective lens and shed on the sample, and FL signals were collected by the same objective lens. The incident wavelength of the continuous-wave laser light was $786.6 \mathrm{~nm}$; the incident laser light went through a line filter for $785 \mathrm{~nm}$ of $2 \mathrm{~nm}$ full width at the half maximum, and the power was $0.5 \mathrm{~mW}$ on the sample surface. In collection path of FL, the scattering of the incident laser light was terminated by sharp-edge and high-optical-density filters that transmit light of wavelengths more than $800 \mathrm{~nm}$. The FL signals traveled through a monochromator and were detected by a charge-coupled device camera. The FL spectra shown in 

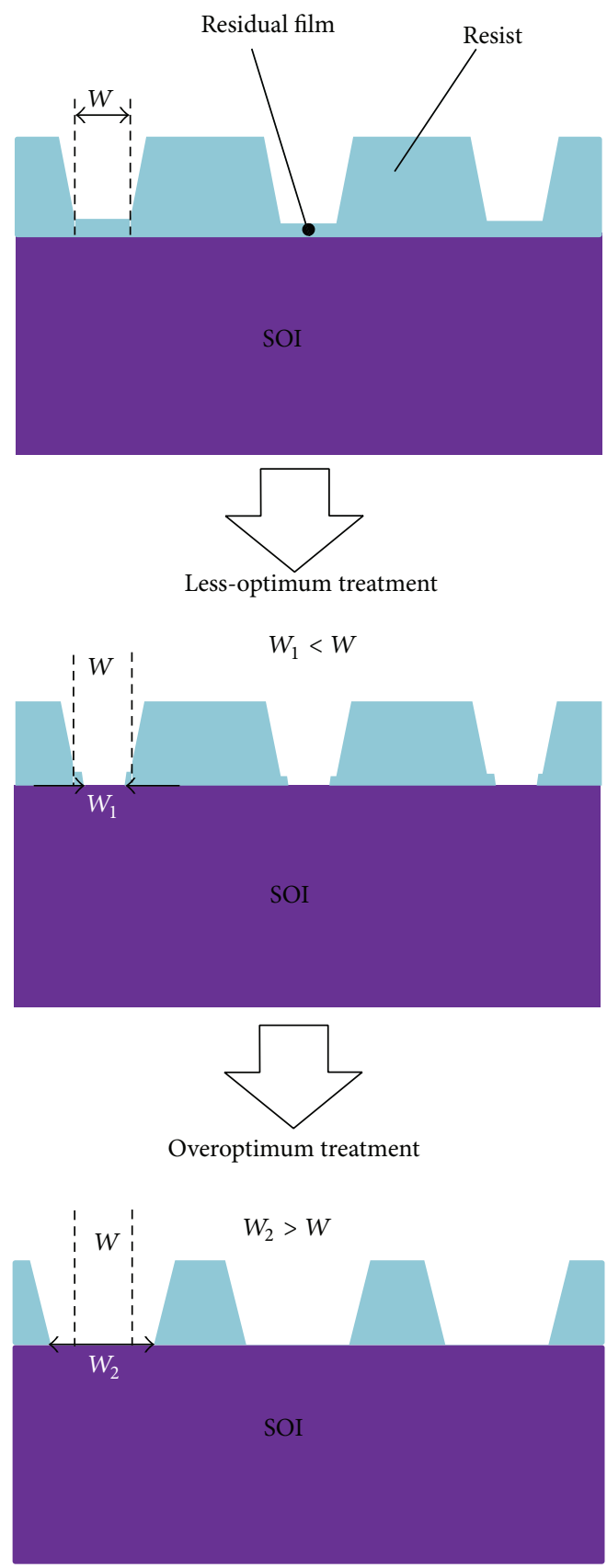

FIGURE 2: Schematic of lateral tuning in NIL. Top: status just after removing the nanoimprint mold. Middle: less-optimum treatment of residual thin film of resist. The aperture width $w_{1}$ is smaller than the initial width $w$. Bottom: overoptimum treatment of residual thin film of resist. The aperture width $w_{2}$ is larger than the initial width $w$. The treatment of the residual film was carried out by dry etching using mixed $\mathrm{O}_{2}$ and $\mathrm{N}_{2}$ gas plasma.

this paper were measured by accumulating the signals for 20 seconds.

For FL measurement at near-infrared range from 800 to $1000 \mathrm{~nm}$, we used dye molecules IR783 (Sigma-Aldrich, St. Louis, USA), whose basic properties are shown in Figure 4. The dye molecules were solved in methanol. The $500 \mu \mathrm{M}$ dye solution was dispersed on the SC metasurface substrate; a drop of $2 \mu \mathrm{L}$ of the dye solution was promptly spread to $18 \mathrm{~mm}$ diameter and became dry. As a result, the dye molecules were dispersed in a quite uniform manner.

In the FL measurement on the SC metasurfaces, before we dispersed the dye molecules, we conducted an additional treatment for the $\mathrm{Au}$ surface to suppress metalinduced quenching of FL in dye molecules that has been often discussed $[11,12]$. Specifically, we made self-assembled monolayer (SAM) on the Au surface; the SAM molecules were 15-carboxy-1-pentadecanethiol (DOJINDO Laboratories, Kumamoto, Japan), grown by following an established protocol [20]. The SAM gap between the Au surface and dispersed dye molecules was estimated to be approximately $1 \mathrm{~nm}$. Note that the SAM may be also formed at the Si side wall in the SC structure.

2.3. Numerical Calculation. We numerically calculated the reflectance spectra and electromagnetic (EM) field distributions by rigorously coupled-wave analysis (RCWA) [21]. To calculate the stacked structures in a numerically stable way, scattering matrix algorithm [22] was incorporated with the RCWA. The author-made computational code was implemented on vector-oriented, 16-CPU supercomputers with a maximum memory of $1.0 \mathrm{~TB}$. We intended to carry out realistic computations; accordingly, permittivity of $\mathrm{Au}$ and Si was taken from literature [23] and [24], respectively, and permittivity of air and $\mathrm{SiO}_{2}$ was set to the representative values, that is, 1.00057 and 2.1316 , respectively.

\section{Results and Discussion}

Figure 3(a) displays the top-view SEM images of the SC metasurfaces fabricated through the UV NIL. The diameter (D) of the air hole was varied from 190 to $288 \mathrm{~nm}$; here we use simple symbols such as D190 to indicate a diameter of $190 \mathrm{~nm}$. Because the diameter of the pillar of the mold was $200 \mathrm{~nm}$ (i.e., $w=200 \mathrm{~nm}$ in Figure 2), it is evident that we obtained both smaller and larger air holes than the $w$ in the UV NIL. Thus, we laterally tuned the structure in a precise manner. Section-view SEM images were also taken and we confirmed the intended SC structure [19].

Figure 3(c) shows a series of reflectance spectra of the SC metasurfaces with $D 190$ to $D 288$. The second resonances in energy, which is excited at incident angle $5^{\circ}$, are labeled as (2) in Figure 3(c). Obviously, the second resonances shift from 1150 to $890 \mathrm{~nm}$ as the diameters increase from D190 to D288. The shift was linearly approximated and found to be $2.7 \mathrm{~nm}$ resonance shift for every $1 \mathrm{~nm}$ air-hole diameter change. Thus, the second resonance covers a wide wavelength range through the UV NIL with only one mold. The reflectance of the second resonance is small and reaches less than $15 \%$ for the SC metasurfaces of D215 and larger air-hole diameters. The small reflectance corresponds to large light absorption of the SC metasurfaces, implying that emittance from the surface is highly efficient. Besides, the width of the reflectance dips is about $30 \mathrm{~nm}$, suggesting a selective enhancement of FL at the resonance band. We therefore test capability of the second resonance for FL enhancement. We mention that 

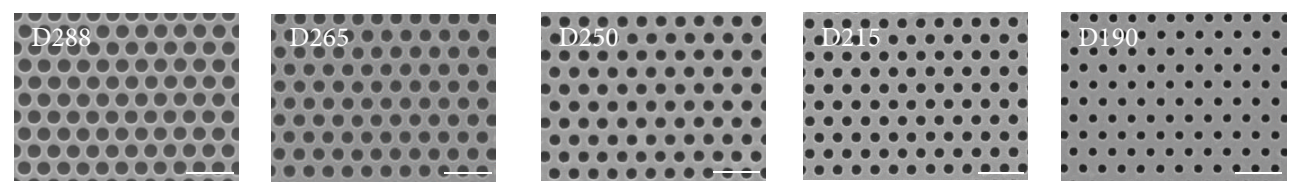

(a)

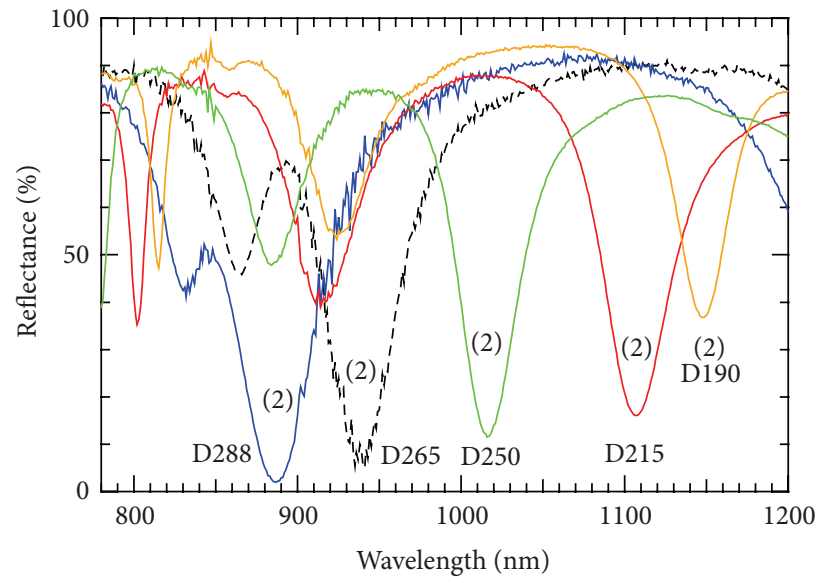

(c)

FIgure 3: (a) Top-view SEM images of the SC metasurfaces with diameter $(D)$ of 288 to $190 \mathrm{~nm}$, which are arrayed from left to right, respectively. The white scale bars indicate $1 \mu \mathrm{m}$. (b) Schematic 3D-view illustration of a SC metasurface and optical configuration. (c) Measured reflectance spectra of the SC metasurfaces at incident angle $\theta=5^{\circ}$ and $p$-polarization in the configuration that the plane of incidence was the $x z$ plane in (b).

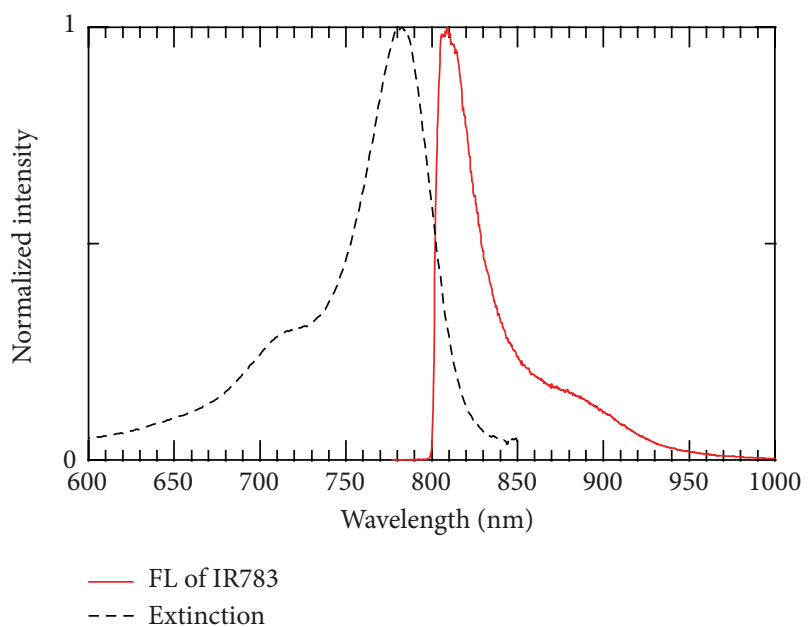

FIGURE 4: Measured FL (red solid line) and extinction (black dashed line) spectra of IR783 in methanol solution. They are normalized at the maximum.

the higher resonances more than the fourth resonance were found to be efficient for FL enhancement in a previous paper [19]. Before describing the details of FL enhancement, we address the basic properties of the IR783 test dye molecules.

Figure 4 shows FL (red solid line) and extinction (black dashed line) spectra of the IR783 dye molecules in methanol solution. The spectra were normalized at the maximum. The peak of the extinction spectrum is located at $783 \mathrm{~nm}$, representing the feature of the dye molecules. The sharp edge of FL at $800 \mathrm{~nm}$ is due to the filters incorporated in the optical measurement setup. The FL rapidly decreases as wavelengths become longer; the FL intensity at $1000 \mathrm{~nm}$ is approximately $1 / 200$ of the peak intensity. We also confirmed that the spectral shape of the FL was similar to that of the FL from the dispersed dye molecules on the $\mathrm{Si}$ and $\mathrm{SiO}_{2}$ substrates.

Figures 5(a) and 5(b) show FL spectra on the SC metasurfaces of D250 and D265, respectively (red); additionally, FL spectra of IR783 on a flat Au film on the nonprocessed SOI substrates are shown with black lines, which are 10 times enlarged for clarity. All the FL spectra were measured under the equivalent condition described in Section 2. The FL signals on the SC metasurfaces were found to be quite larger than the FL signals on the flat Au surface. The reflectance spectra of the SC metasurfaces are also shown with gray dashed lines in Figures 5(a) and 5(b). The FL spectra on the SC metasurfaces have peaks around the reflectance dips of the second (2) and third (3) resonances.

Figure 5(c) shows FL-intensity ratio at the peak of the FL spectra in Figures 5(a) and 5(b). The ratio was simply taken as the ratio of FL intensity on the SC metasurfaces to that on the flat Au surface; the cases of D250 and D265 are shown with red and green, respectively. The FL-intensity ratio exceeds 450 at $990 \mathrm{~nm}$ in the case of D250, exhibiting large FL enhancement effect. Also, the FL-intensity ratio is larger than 100 at $915 \mathrm{~nm}$ in the case of D265. Note that FL enhancement factor (EF) is usually discussed in enhanced FL studies [7, 8]; the FL EF is well-defined using a relevant reference that has 


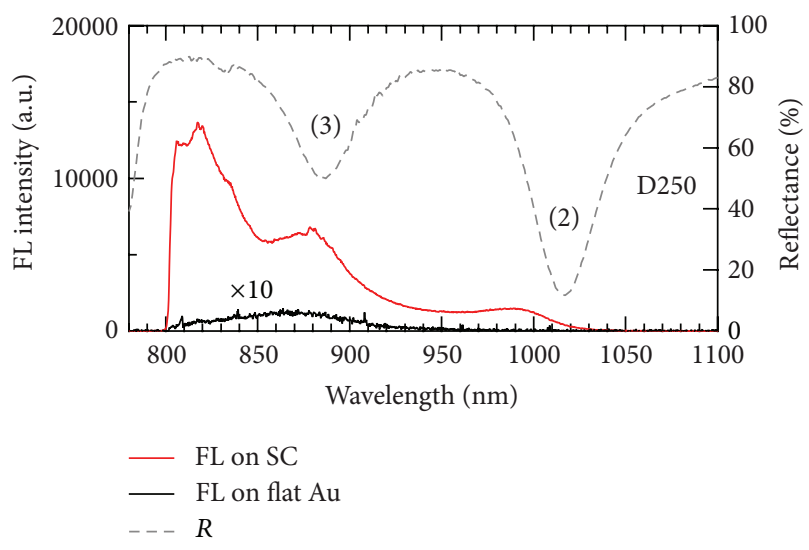

(a)

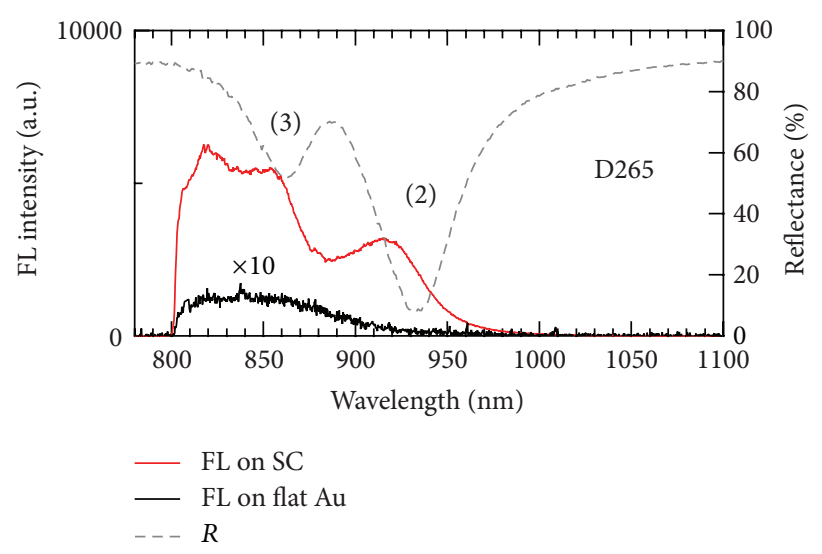

(b)

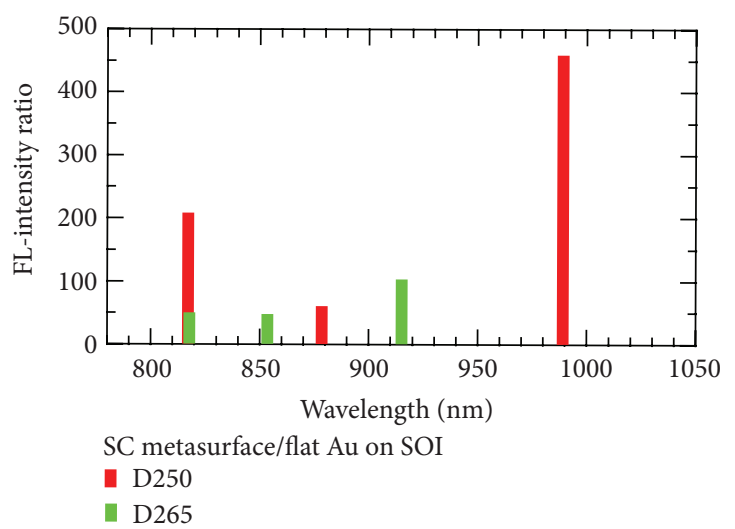

(c)

Figure 5: (a) FL spectra of IR783 on the SC metasurface of D250 (red) and on the flat Au on the SOI substrate (black). The latter was 10 times enlarged for clarity. The reflectance $(R)$ spectrum (gray dashed line) is plotted for the right axis. The second and third resonances are indicated by (2) and (3), respectively. (b) FL spectra on the SC metasurface of D265 and on the flat Au on the SOI substrate are shown similar to (a). (c) FL-intensity ratio, which was evaluated by dividing the FL intensity on SC metasurface by the FL intensity on the flat Au on the SOI substrate. Red bars correspond to D250 and green bars to D265.

similar surface absorption property for the test molecules. In the present cases, we could not prepare an ideal reference such as flat $\mathrm{Au}$ film on $\mathrm{Si}$ or $\mathrm{SiO}_{2}$ substrates because FL signals on the references were very weak and it was difficult to take the FL ratio. Therefore, we had to replace the candidates for ideal reference with the flat $\mathrm{Au}$ film on the SOI substrate; the FL signals on the flat Au film on the SOI substrate tend to be larger than the ideal reference, resulting in reduction of FL-intensity ratio. Summing up the considerations above, we can experimentally conclude that the FL of the IR783 was enhanced by more than 450 times on the second resonance at about $1 \mu \mathrm{m}$. Thus, the second resonance is also efficient for FL enhancement, similar to the higher resonances [19].

Figure 6 presents numerically calculated EM field distributions on the second resonance. The structural parameters were taken from the SC metasurface of D250. Figures 6(a) and 6(b) show the absolute values of electric field $|\mathrm{E}|$ on the top and bottom layers, respectively, in the $x y$ plane (see Figure 3 (b) for the coordinate), where most of the dispersed dye molecules are expected to be located. Strictly, the $x y$ planes at $1 \mathrm{~nm}$ above from the top and bottom layers are plotted because the SAM in experiment was approximately $1 \mathrm{~nm}$ thickness. In Figure 6(a), the positions of the air holes are shown with circles. The incidence was set to be $x$-polarized, to shed normally on the $x y$ plane, and to have $|\mathbf{E}|=1$. On the top layer, the most enhanced electric fields appear at the edge of the air holes and take the maximum value of 3.3. On the bottom layer, air domain, where the dispersed dye molecules are located, is limited to inside the air holes, and the most intense electric fields appear at the very rim of the air holes, taking the maximum value of 7.1. Thus the so-called hot spots were observed whereas the spots are limited to very narrow width less than $10 \mathrm{~nm}$ for $|\mathbf{E}|>5$; consequently, the portion of the dye molecules dispersed at the hot spots is considered to be very small. Overall, the averaged electric field intensity $|\mathbf{E}|^{2}$ was estimated to be less than 5; therefore, the FL transition was 5-fold expedited on average by the electric field intensity on the SC metasurfaces. This factor is too small for the observed FL-intensity ratio more than 450 . At the excitation wavelength, enhancement due to the electric 


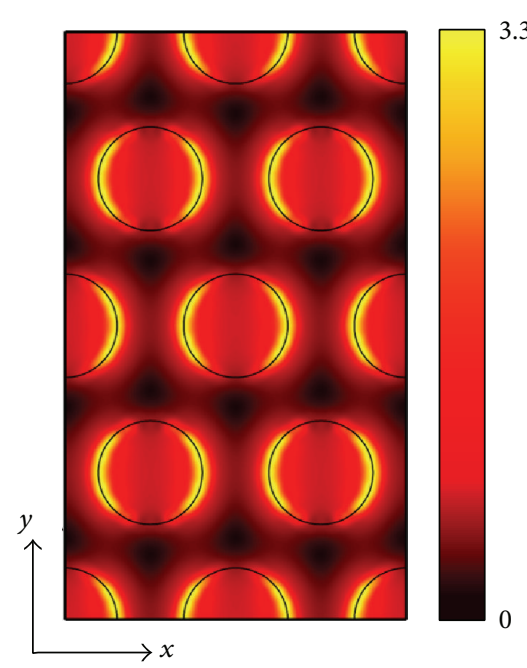

(a)

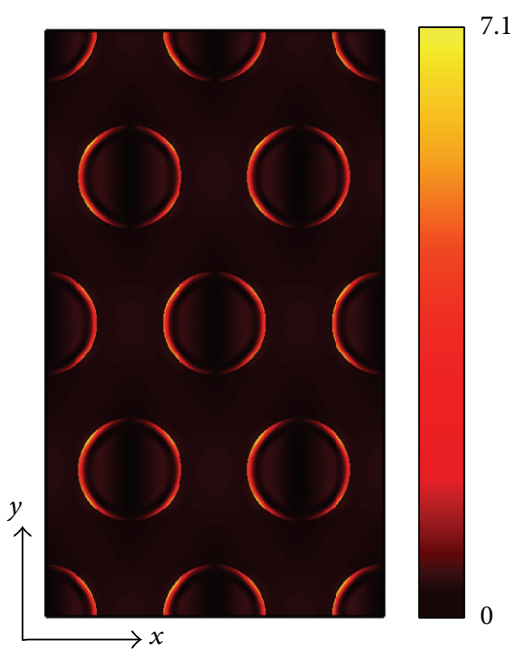

(b)

FIGURE 6: Numerically calculated absolute values of electric field distributions $|\mathbf{E}|$ on the second resonance in the $x y$ plane, corresponding to the SC metasurface of D250. (a) and (b) $|\mathbf{E}|$ at $1 \mathrm{~nm}$ above from the top and bottom layers, respectively. The incidence was set to have $|\mathbf{E}|=1$.

field intensity is also expected; however, the average intensity is not large enough to account for the FL-intensity ratio in Figure 5(a). In general, FL EF is expressed as $[8,25]$

$$
\mathrm{EF}=\frac{\eta}{\eta_{0}} \frac{\gamma_{r}(\mathbf{k})}{\gamma_{0}(\mathbf{k})}
$$

where $\eta_{0}$ and $\gamma_{0}$ are quantum yield of FL and radiative transition rate in reference circumstance, respectively; $\mathbf{k}$ denotes outgoing wavevector of $\mathrm{FL} ; \gamma_{r}$ denotes radiative transition rate in enhanced circumstance; $\eta$ is radiative transition rate in enhanced circumstance and written as $\eta=N_{\text {exc }} \gamma_{r} /\left(\gamma_{r}+\right.$ $\gamma_{\mathrm{nr}}$ ) where $N_{\mathrm{exc}}$ is excited population per second and $\gamma_{\mathrm{nr}}$ is nonradiative transition rate in enhanced circumstance. The electric field intensity at the excitation wavelength is proportional to $N_{\text {exc }}$. Note that the EF depends on $\gamma_{r}$ in a nonlinear way and can be significantly large when the ratio of $\gamma_{r} / \gamma_{\mathrm{nr}}$ changes. The results in Figure 5(c) suggest the change in electronic transition rates in the enhanced circumstance.

As for the large EF reported so far, narrow gaps less than $5 \mathrm{~nm}$ between metallic nanostructures were sought for and EFs more than 1000 were reported $[9,10]$. As is clear in (1), EF depends on the test molecules themselves because it includes the quantum yield $\eta_{0}$. Thus it is improper to compare EFs using different test molecules. We here mention that the present FL-enhancing results are more uniform than the previous reports $[9,10]$ because FL-intensity fluctuations were approximately $5 \%$ in our measurement and that detecting wavelengths were now near-infrared around $1 \mu \mathrm{m}$ and were narrowband because the second resonance has about $30 \mathrm{~nm}$ line width. These features have not been reported to date. The present enhancement capability at the narrow bands will be useful to meet the demand for selective detection of enhanced FL and Raman signals.

FL enhancement on higher resonances in $\mathrm{Si}$ photonic crystal slabs was recently reported [26]. Although the dye molecules were Rhodamine series different from the present
IR783, FL enhancement factor of about 250 was obtained. The SC metasurfaces have advantages in flexible resonance tuning at a wide wavelength range in the near-infrared and in cost-effective NIL with only one mold. If one needs to change resonant wavelengths in Si photonic crystal slabs, it would be necessary to change the periodicity and to obtain expensive mold(s). On the other hand, Si photonic crystal slabs are chemically and thermally stable, compared to the SC metasurfaces. Thus the two platforms have different advantages.

\section{Conclusions}

We have implemented lateral structure tuning in UV NIL with only one mold and successfully produced a series of the large-area SC metasurfaces with different air-hole diameters. The series exhibited a systematic resonance shift at a wide near-infrared range. Furthermore, the second resonance in the SC metasurfaces was employed to realize FL enhancement in the near-infrared range around $1 \mu \mathrm{m}$ and enabled us to observe a large FL-intensity ratio more than 450 on a SC metasurface in comparison with a flat Au film on the SOI substrate. The resonant EM fields were numerically calculated and discussed in terms of the contribution of the electric field intensity to the large FL-intensity ratio. The SC metasurfaces are capable of selectively enhancing FL signals at the narrow band of $30 \mathrm{~nm}$ and of serving as platforms for on-demand enhanced spectroscopy.

\section{Conflict of Interests}

All authors have no conflict of interests to declare.

\section{Acknowledgments}

This study was partially supported by a Grant-in-Aid for Scientific Research on Innovative Areas (no. 22109007) from 
the Ministry of Education, Cultures, Sports, Science and Technology, Japan. Masanobu Iwanaga also acknowledges the support by a Grant-in-Aid from Japan Society for the Promotion of Science (no. 26706020) and the support for numerical implementations at Cyberscience Center, Tohoku University, and Cybermedia Center, Osaka University, through HPCI System Research Project (ID hp140068). Nanofabrications and the characterizations of the SC metasurfaces were conducted at Nano-Integration Foundry and Low-Carbon Research Network in NIMS.

\section{References}

[1] J. B. Pendry and D. R. Smith, "Reversing light with negative refraction," Physics Today, vol. 57, no. 6, pp. 37-43, 2004.

[2] V. M. Shalaev, "Optical negative-index metamaterials," Nature Photonics, vol. 1, no. 1, pp. 41-48, 2007.

[3] C. M. Soukoulis and M. Wegener, "Past achievements and future challenges in the development of three-dimensional photonic metamaterials," Nature Photonics, vol. 5, no. 9, pp. 523-530, 2011.

[4] N. I. Zheludev and Y. S. Kivshar, "From metamaterials to metadevices," Nature Materials, vol. 11, no. 11, pp. 917-924, 2012.

[5] M. Iwanaga, "Photonic metamaterials: a new class of materials for manipulating light waves," Science and Technology of Advanced Materials, vol. 13, no. 5, Article ID 053002, 2012.

[6] N. Yu, P. Genevet, M. A. Kats et al., "Light propagation with phase discontinuities: generalized laws of reflection and refraction," Science, vol. 334, no. 6054, pp. 333-337, 2011.

[7] J. R. Lakowicz, K. Ray, M. Chowdhury et al., "Plasmon-controlled fluorescence: a new paradigm in fluorescence spectroscopy," Analyst, vol. 133, no. 10, pp. 1308-1346, 2008.

[8] M. Bauch, K. Toma, M. Toma, Q. Zhang, and J. Dostalek, "Plasmon-enhanced fluorescence biosensors: a review," Plasmonics, vol. 9, no. 4, pp. 781-799, 2014.

[9] A. Kinkhabwala, Z. Yu, S. Fan, Y. Avlasevich, K. Müllen, and W. E. Moerner, "Large single-molecule fluorescence enhancements produced by a bowtie nanoantenna," Nature Photonics, vol. 3, no. 11, pp. 654-657, 2009.

[10] W. Zhang, F. Ding, W.-D. Li, Y. Wang, J. Hu, and S. Y. Chou, "Giant and uniform fluorescence enhancement over large areas using plasmonic nanodots in 3D resonant cavity nanoantenna by nanoimprinting," Nanotechnology, vol. 23, no. 22, Article ID 225301, 2012.

[11] S. C. Kitson, W. L. Barnes, and J. R. Sambles, "Surface-plasmon energy gaps and photoluminescence," Physical Review B, vol. 52, no. 15, pp. 11441-11445, 1995.

[12] P. Anger, P. Bharadwaj, and L. Novotny, "Enhancement and quenching of single-molecule fluorescence," Physical Review Letters, vol. 96, no. 11, Article ID 113002, 2006.

[13] S. Y. Chou, P. R. Krauss, and P. J. Renstrom, "Imprint of sub-25 $\mathrm{nm}$ vias and trenches in polymers," Applied Physics Letters, vol. 67, no. 21, pp. 3114-3116, 1995.

[14] S. Y. Chou, P. R. Krauss, W. Zhang, L. Guo, and L. Zhuang, "Sub$10 \mathrm{~nm}$ imprint lithography and applications," Journal of Vacuum Science and Technology B: Microelectronics and Nanometer Structures, vol. 15, no. 6, pp. 2897-2904, 1997.

[15] L. J. Guo, "Nanoimprint lithography: methods and material requirements," Advanced Materials, vol. 19, no. 4, pp. 495-513, 2007.
[16] Y. Ofir, I. W. Moran, C. Subramani, K. R. Carter, and V. M. Rotello, "Nanoimprint lithography for functional three-dimensional patterns," Advanced Materials, vol. 22, no. 32, pp. 36083614, 2010.

[17] Y. Fukuda, Y. Saotome, N. Nishiyama et al., "Fabrication of nanodot array mold with $2 \mathrm{Tdot} / \mathrm{in}$. $^{2}$ for nanoimprint using metallic glass," Journal of Vacuum Science and Technology B: Microelectronics \& Nanometer Structures, vol. 30, no. 6, Article ID 061602, 2012.

[18] X. M. Yang, S. Xiao, Y. Hsu, M. Feldbaum, K. Lee, and D. Kuo, "Directed self-assembly of block copolymer for bit patterned media with areal density of 1.5 Teradot/Inch ${ }^{2}$ and beyond," Journal of Nanomaterials, vol. 2013, Article ID 615896, 17 pages, 2013.

[19] B. Choi, M. Iwanaga, H. T. Miyazaki, K. Sakoda, and Y. Sugimoto, "Photoluminescence-enhanced plasmonic substrates fabricated by nanoimprint lithography," Journal of Micro/Nanolithography, MEMS, and MOEMS, vol. 13, no. 2, Article ID 023007, 2014.

[20] http://www.dojindo.com/Shared/Protocol/SAM_Protocol.pdf.

[21] L. Li, "New formulation of the Fourier modal method for crossed surface-relief gratings," Journal of the Optical Society of America A, vol. 14, no. 10, pp. 2758-2767, 1997.

[22] L. Li, "Formulation and comparison of two recursive matrix algorithms for modeling layered diffraction gratings," Journal of the Optical Society of America A: Optics and Image Science, and Vision, vol. 13, no. 5, pp. 1024-1035, 1996.

[23] A. D. Rakić, A. B. Djurišić, J. M. Elazar, and M. L. Majewski, "Optical properties of metallic films for vertical-cavity optoelectronic devices," Applied Optics, vol. 37, no. 22, pp. 5271-5283, 1998.

[24] E. D. Palik, Handbook of Optical Constants of Solids II, Academic Press, San Diego, Calif, USA, 1991.

[25] L. Novotny and B. Hecht, Principles of Nano-Optics, Cambridge University Press, New York, NY, USA, 2nd edition, 2012.

[26] B. Choi, M. Iwanaga, T. Ochiai, H. T. Miyazaki, Y. Sugimoto, and K. Sakoda, "Subnanomolar fluorescent-molecule sensing by guided resonances on nanoimprinted silicon-on-insulator substrates," Applied Physics Letters, vol. 105, no. 20, Article ID 201106, 2014. 

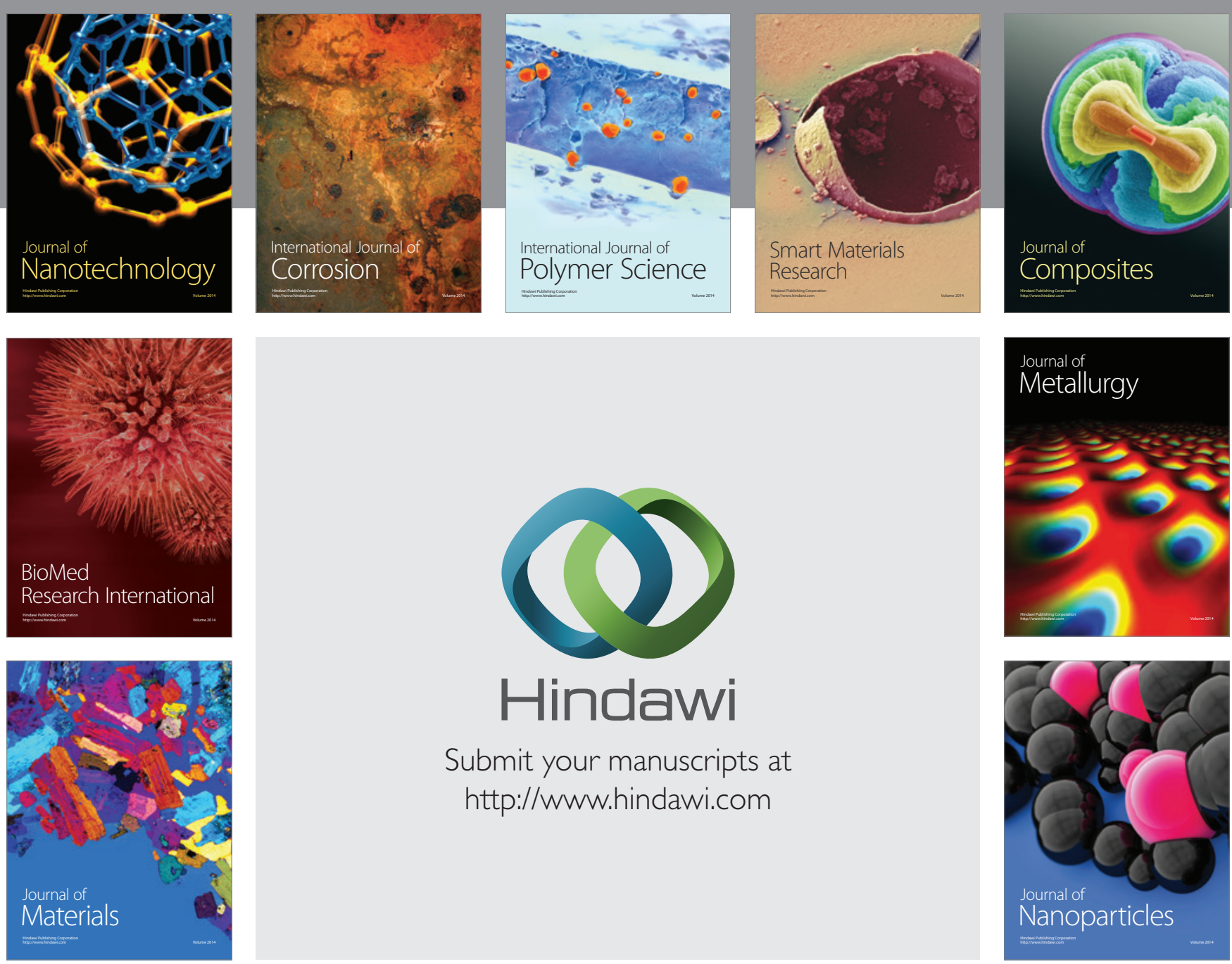

Submit your manuscripts at http://www.hindawi.com
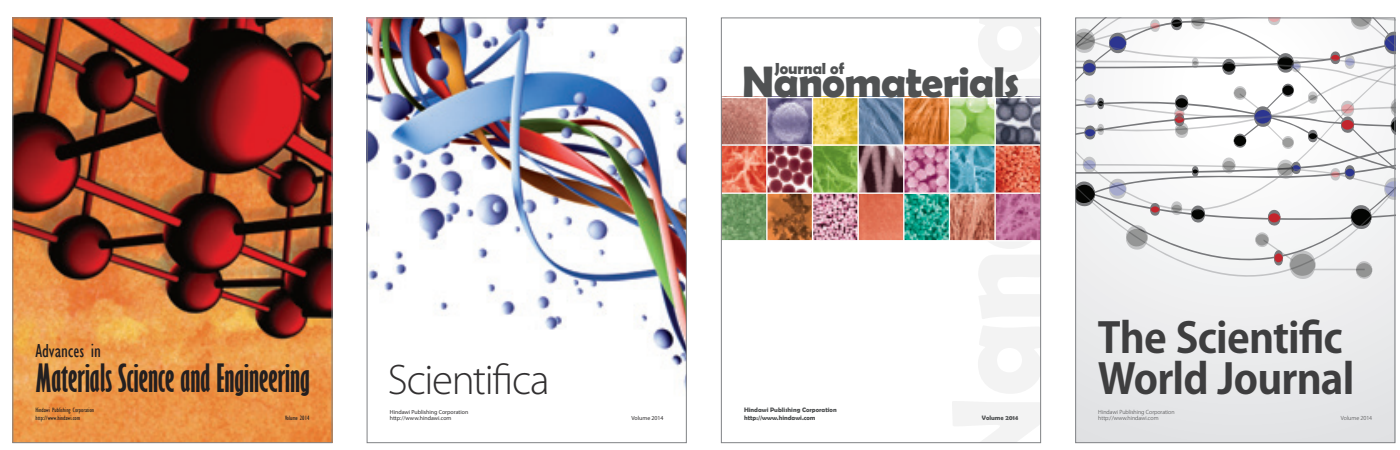

\section{The Scientific World Journal}
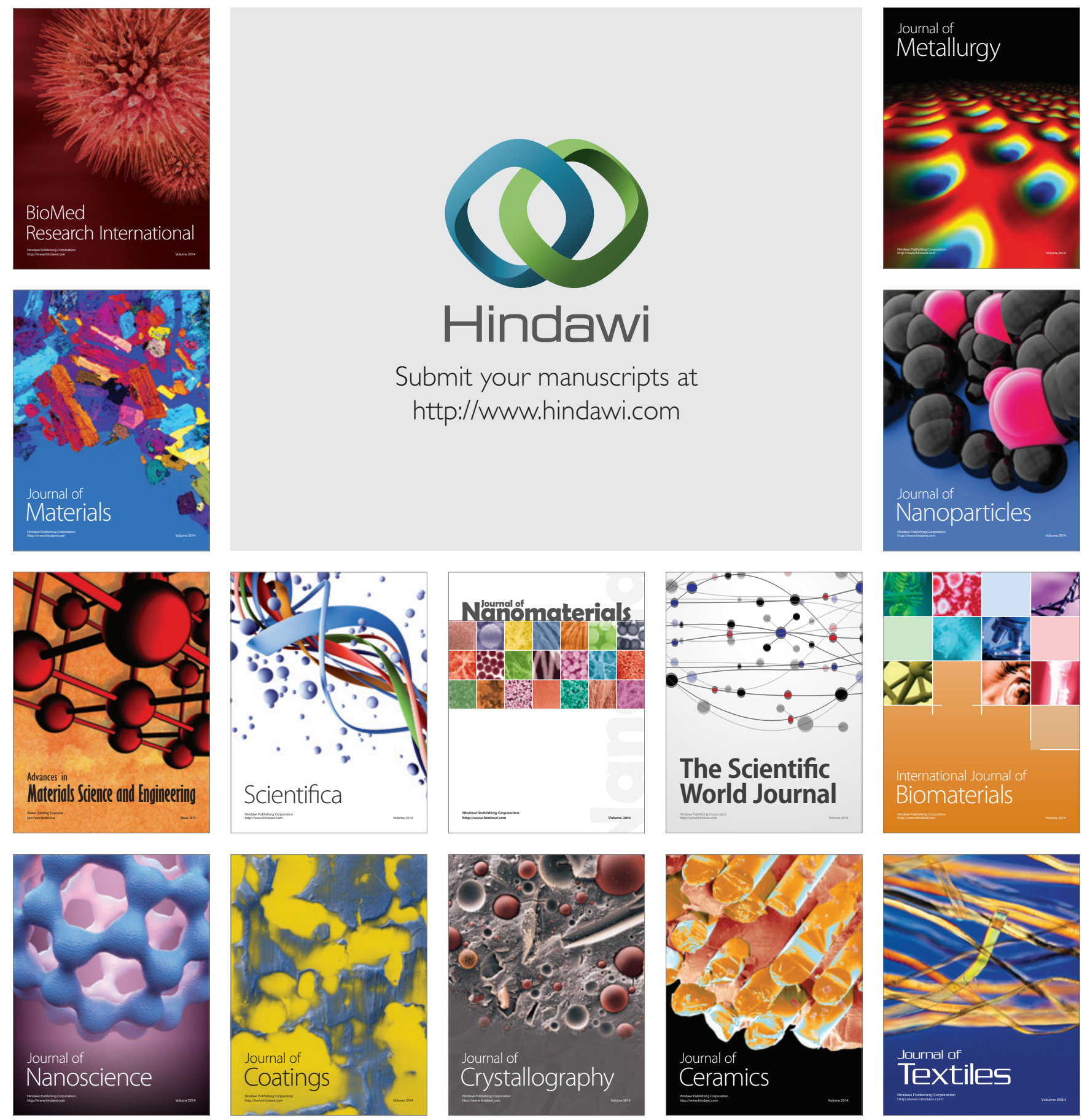